\title{
45 Special features of diets
}

Statistically, people in the industrialized countries consume between 130-150 g fat every day, whereas only around $90-110 \mathrm{~g}$ is justifiable from a long-term health perspective, depending on the individual's caloric needs. However, for many, it is often difficult sticking to within these limits. Indeed, many foodstuffs or cafeteria food frequently contain fat content that is not listed on the label. And a load of fat is hidden where you would least expect it, i. e. as much as up to $8 \%$ in whole grain bread. But not only do we eat too much fat, we mostly eat animal fat, i. e. the wrong kind of fat that contains saturated fatty acids.

$10 \mathrm{~g}$ fat (saturated fatty acids) are contained in:

- $2 / 3$ croissant

- 2 yogurt bars (25 g)

- 16 potato chips

- 20 g roasted peanuts

- 20 g chocolate

- 32 g whipping cream

- 4 cups of mélange coffee

Too high fat intake can cause overweight. Unfortunately, the diets that invariably follow are usually associated with problems of malnutrition.

In this context, for instance, diets are constantly propagated that allow unlimited fat consumption, but reduce consumption of carbohydrates in compensation. From the perspective of losing weight, this might appear to constitute a rather promising strategy at first glance. This ties in with the fact that most people tend to dislike eating large amounts of fat without consuming them simultaneously with carbohydrates, thereby also very reliably lowering their energy uptake. This leads to quick initial success, which is how the likes of the Atkins diet earned their popularity. All large studies on this topic have shown, however, that weight loss from a diet lasting a year and longer is solely dictated by the duration and the restriction of calories and not from the choice of energy transfer media.
Irrespective of the above, fat-rich diets are always harmful due to the unbridled uptake of saturated fatty acids and the associated elevation in cholesterol biosynthesis.

Effective weight reduction programs always go hand-in-hand with a sensible change in nutritionrelated habits. This includes a balanced diet consisting of abundant complex carbohydrates e. g. in fruits, vegetables, potatoes and grains. These are carriers of minerals, trace elements, bioactive plant compounds, dietary fiber and most vitamins. In this context, note should be taken of the fact that, unlike complex carbohydrates, simple carbohydrates in the form of sugar are mere energy suppliers. The daily intake of sugar, e.g. from honey, jam, sweet baked goods, sweet drinks, chocolate, other sweets or hidden in various convenience meals and/or food additives, should therefore be limited to a maximum of $10 \%$ (approx. $50-75 \mathrm{~g}$ sugar) of the respective recommended daily energy intake (WHO 2009). 